\title{
Microwave Losses of Interferometers with many Josephson Junctions versus DC Magnetic Field
}

\author{
R. Lochschmied, W. Benzing and W. Jutzi \\ Institut für Elektrotechnische Grundlagen der Informatik, University of Karlsruhe, D-76187 Karlsruhe
}

\begin{abstract}
The average microwave losses of interferometers with two and four strongly damped Josephson junctions biased by an microwave current source at $10 \mathrm{GHz}$ much below the plasma frequency have been simulated as a function of an external magnetic field. Microwave losses appear periodically within distinct ranges of the magnetic field, with microwave amplitudes below and above threshold currents. The main period corresponds to one flux quantum in every interferometer loop. The absorption rate increases with microwave power in qualitative agreement with measurements. As the microwave has a finite penetration depth from one end of a long interferometer with many Josephson junctions, the absorption lines decrease within the main period.
\end{abstract}

\section{INTRODUCTION}

One dimensional arrays of Josephson junctions can be used to explain electrical and magnetical effects in superconductor devices. The magnetic sensitivity of the microwave absorption in a single $\mathrm{YBa}_{2} \mathrm{Cu}_{3} \mathrm{O}_{7-\delta}$ crystal can be modeled by an interferometer with many Josephson junctions. The absorption of microwave power was calculated in [1] using a superconductor loop with many junctions. Another method was proposed in [2], based on a model of weakly coupled superconducting loops.

In this work the microwave losses have been simulated with the circuit analysis program SPICE. A simple RCSJ-model (McCumber) with a nonlinear quasiparticle current, has been compared with a more complex simulation model, based on the theory of Werthamer (WA-model) [3]. The interferometer simulations assume a critical current density of $j_{\max }=0.52 \mathrm{kA} / \mathrm{cm}^{2}$. The junction capacitance is $\mathrm{C}_{\mathrm{F}}=5 \mu \mathrm{F} / \mathrm{cm}^{2}$, corresponding to a plasma frequency

$$
f_{p}=\sqrt{\frac{j_{\max }}{2 \pi \cdot \Phi_{0} \cdot C_{F}}}=89.5 \mathrm{GHz} \text {. }
$$

The junction area of a single point junction is $\mathrm{A}=20 \mu \mathrm{m}^{2}$, the critical current $\mathrm{I}_{0}=0.1 \mathrm{~mA}$ and the capacitance of a point junction $\mathrm{C}_{\mathrm{J}}=1 \mathrm{pF}$. The Josephson junctions are strongly damped with a shunt resistor $\mathrm{R}$ to get a nonhysteretic I,V-characteristic similar to a SNS junction. The McCumber parameter of a Josephson junction is

$$
\beta=\frac{2 \pi}{\Phi_{0}} \cdot R_{F}^{2} \cdot C_{F} \cdot j_{\max }=1.3
$$

with $\mathrm{R}=2 \Omega$ and $\mathrm{R}_{\mathrm{F}}=\mathrm{R} \cdot \mathrm{A}=40 \Omega \mu \mathrm{m}^{2}$.

Manuscript received October 16, 1994, ASC '94 Boston
Both simulation models have the same quasiparticle $\mathrm{I}, \mathrm{V}$-curve in a sufficient large magnetic field. The average power dissipation versus frequency has been calculated. With the above parameters, no essential difference between the results of WA- and RCSJ-model at $4.2 \mathrm{~K}$ and a frequency of $\mathrm{f}_{\mathrm{LO}}=10 \mathrm{GHz}$ has been found, where the microwave absorption in a single $\mathrm{YBa}_{2} \mathrm{Cu}_{3} \mathrm{O}_{7-\delta}$ crystal has been measured [4]-[6]. Since the WA-model needs more computation time, the RCSJ-model has been used to get the results of this paper. The resistors are assumed to have no Johnson noise.

\section{MICROWAVE LOSSES IN A SQUID}

An interferometer with two identical strongly damped Josephson junctions is chosen as a simple model to describe approximately microwave losses in a single $\mathrm{YBa}_{2} \mathrm{Cu}_{3} \mathrm{O}_{7-\delta}$ crystal. With the mentioned parameters and a inductance $\mathrm{L}=4.97 \mathrm{pH}$ the characteristic phase is

$$
\lambda=\frac{2 \pi}{\Phi_{0}} \cdot L \cdot I_{0}=0.1 \pi \text {. }
$$

The microwave is injected in the center of the inductance. The control current $I_{c}=\Phi_{C} / L$ represents the DC magnetic field. The circuit diagram of the simulated SQUID is sketched in Fig. 1.

The static SQUID characteristics [7],[8] with different flux modes, corresponding to $0,1,2$ and 3 flux quanta in the loop, are plotted in Fig. 2a. The static SQUID characteristic is periodical with $\Phi_{0}$. The flux modes are stable inside the closed curves. Outside the threshold characteristic the SQUID switches into the voltage state.

The injected microwave current has a sinusoidal shape $I=\hat{I}_{L O} \cdot \sin \left(2 \pi f_{L O} t\right)$ at a frequency $\mathrm{f}_{\mathrm{LO}}=10 \mathrm{GHz}$ far below the plasma frequency $f_{p}$. The control current is kept constant

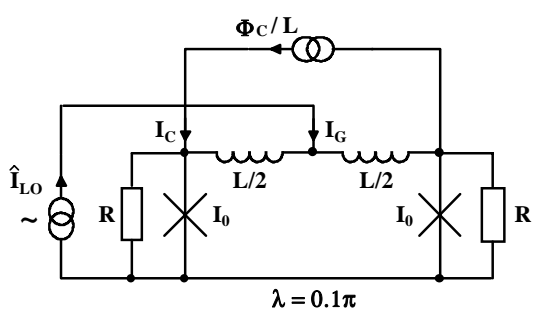

Fig. 1. Circuit diagram of the simulated SQUID. Normalized parameters are $\lambda=0.1 \pi$ and $\beta=1.3$. The microwave current is symmetrically injected at the gate. The magnetic field is modeled by the DC-current $\Phi_{\mathrm{C}} / \mathrm{L}$. 
for every calculation of the power losses. The average of the instantaneous power

$$
p=U(t) \cdot I_{L O} \cdot \sin \left(2 \pi f_{L O} t\right)
$$

is numerical taken over ten microwave periods. The computation of the average starts, after the system has reached the steady state. Each absorption curve in Fig. 2b-d comprises 600 simulated points for $\Phi_{\mathrm{C}}$. The average power is normalized with the power dissipation in the shunt resistors

$$
p_{N}=\frac{1}{2} \cdot \frac{R}{N} \cdot \hat{I}_{L O}^{2}
$$

The normalized power dissipation of the SQUID $\bar{p} / p_{2}$ for different normalized microwave amplitudes $\hat{I}_{L O} / I_{0}$ is plotted in Fig. 2. For $\hat{I}_{L O} / I_{0}=0.06$ the microwave current does not exceed the threshold characteristic from a stable flux mode to the voltage state at any point of $\Phi_{C}$. Nevertheless, maxima of power dissipation appear periodically, when the microwave current gets close to the threshold current.

The first derivation $\mathrm{s}=\mathrm{dp} / \mathrm{d} \Phi_{\mathrm{C}}$ calculated with the results in Fig. 2b,c and $d$ reminds microwave absorption measurements at single $\mathrm{YBa}_{2} \mathrm{Cu}_{3} \mathrm{O}_{7-\delta}$ crystals [4]-[6]. The linewidth $\Phi_{\mathrm{B}}$ is introduced as the difference of two values $\Phi_{\mathrm{C}}$ between the highest positive and negative slope, i. e. $\left|s_{\max }\right|$. For microwave currents larger than the thresholds in Fig. 2a the SQUID switches into the "voltage state", as shown in

a)

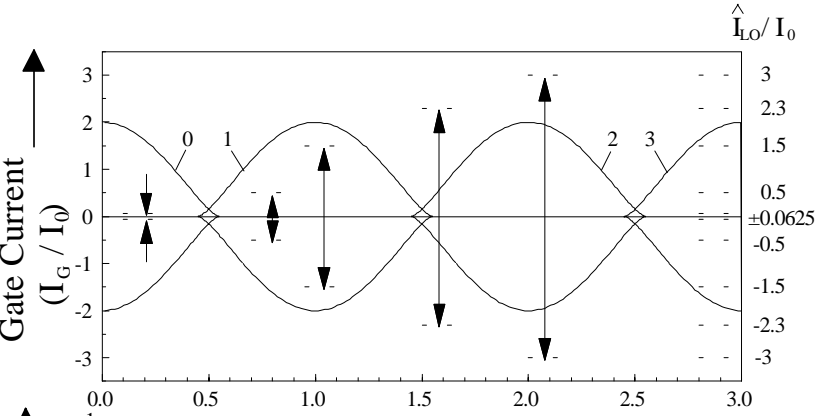

b)

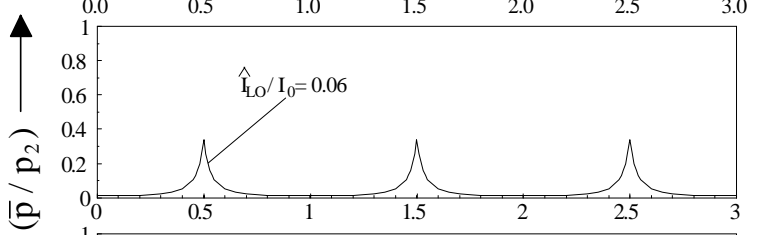

c)

d)

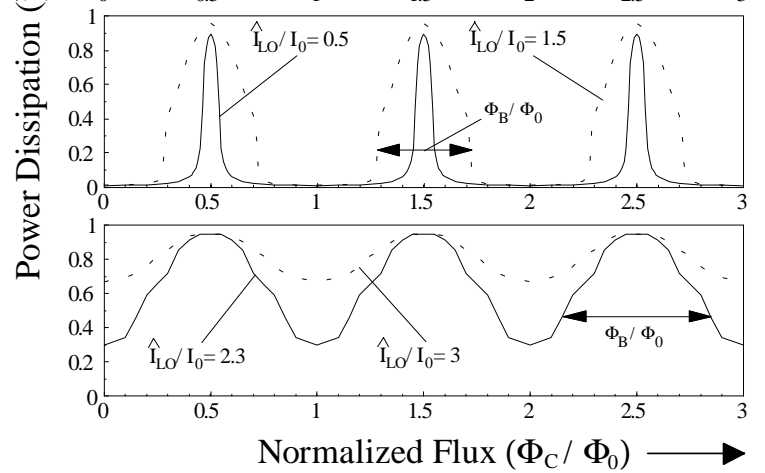

Fig. 2. normalized power dissipation of a SQUID with $\lambda=0.1 \pi$ versus flux. a) Static interferometer characteristics of a SQUID. plotted are the flux modes $0,1,2$ and 3 .

Average power dissipation at the microwave amplitude $\begin{array}{ll}\text { b) } I_{L O}=0.06 \cdot I_{0} & \text { c) } I_{L O}=0.5 \cdot I_{0} \text { and } I_{L O}=1.5 \cdot I_{0}\end{array}$ d) $\hat{I}_{L O}=2.3 \cdot I_{0}$ and $\hat{I}_{L O}=3 \cdot I_{0}$

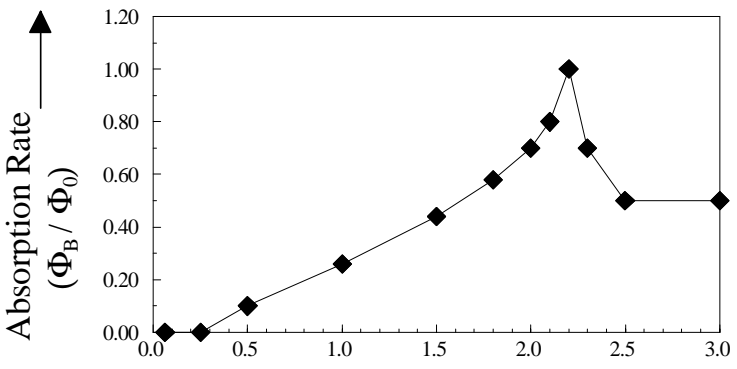

Microwave Amplitude $\left(\mathrm{I}_{\mathrm{LO}} / \mathrm{I}_{0}\right) \longrightarrow$

Fig. 3. Normalized linewidth of the SQUID $\Phi_{\mathrm{B}} / \Phi_{0}$ versus normalized microwave amplitude.

Fig. 2c, where the phases rotate in one direction during one half of the microwave period, and dissipates considerably more power than in the "non voltage state". Simulations with different amplitudes $\hat{I}_{L O}=0.5 \cdot I_{0}$ and $\hat{I}_{L O}=1.5 \cdot I_{0}$ illustrate that the linewidth broadens with increasing microwave amplitude.

If the amplitude increases further, the SQUID will remain in the quasi stable voltage state at any value of $\Phi_{\mathrm{C}}$ as shown in Fig. $2 \mathrm{~d}$ for $\hat{I}_{L O}=2.3 \cdot I_{0}$ and $\hat{I}_{L O}=3 I_{0}$. With increasing microwave current, the dependence on $\Phi_{C}$ changes from sharp peaks to a smooth sinusoidal function.

The normalized linewidth $\Phi_{\mathrm{B}} / \Phi_{0}$ versus microwave amplitude is shown in Fig. 3. If the microwave is below the threshold current, the linewidth $\Phi_{\mathrm{B}}$ is extremely small. A threshold of $\hat{I}_{L O} / I_{0}$ could be defined. With increasing amplitude the SQUID switches into the voltage state. If $\hat{I}_{L O}<2 I_{0}$, the linewidh increases almost linearly. At $\hat{I}_{L O}>2 I_{0}$ the normalized linewidth increases rapidly to its maximum, then it falls to its saturation value of $\Phi_{\mathrm{B}} / \Phi_{0}=0.5$. The increase of the linewidth is in qualitative agreement with measurements [5],[9].

\section{DYNAMIC EFFECTS IN LONG JOSEPHSON JUNCTIONS}

A single SQUID is an unsuitable model to describe the microwave penetration into a single $\mathrm{YBa}_{2} \mathrm{Cu}_{3} \mathrm{O}_{7-\delta}$ crystal. A uniform long junction might give a better insight of basic properties. To get a simple approximation, small variations of $\varphi$ in the nonstationary Sine Gordon equation with damping are assumed for a replacement of the $\sin (\varphi)$-term by $\varphi$. With the complex microwave phase $\varphi=\hat{\varphi}_{L O} \cdot e^{j 2 \pi f_{L O} t}$ and after some algebraic transformations the simple differential equation is obtained

$$
\frac{\partial^{2} \varphi}{\partial x^{2}}-\frac{1}{\lambda_{J}^{2}}\left|1-\left(\frac{f_{L O}}{f_{P}}\right)^{2}+j \frac{f_{L O}}{f_{c}}\right| \cdot \varphi \approx 0
$$

The Josephson penetration depth is

$$
\lambda_{J}=\sqrt{\frac{\Phi_{0}}{2 \pi \mu_{0} \cdot d \cdot j_{\max }}},
$$


with $\mathrm{d}=\mathrm{t}_{\mathrm{ox}}+\lambda_{\mathrm{L} 1}+\lambda_{\mathrm{L} 2}$. The London penetration depths of the junction electrodes are $\lambda_{\mathrm{L} 1}$ and $\lambda_{\mathrm{L} 2} \cdot \mathrm{t}_{\mathrm{ox}}$ is the layer thickness between both electrodes, and $f_{p}$ is the plasma frequency of the junction. The characteristic frequency is

$$
f_{c}=\frac{I_{0} \cdot R}{\Phi_{0}} .
$$

With $\mathrm{f}_{\mathrm{p}}>\mathrm{f}_{\mathrm{LO}} \approx \mathrm{f}_{\mathrm{c}}$ the differential equation

$$
\frac{\partial^{2} \varphi}{\partial x^{2}}-\frac{1}{\lambda_{J}^{2}} \cdot(1+j) \cdot \varphi \approx 0
$$

indicates, that microwave losses modify the penetration of the magnetic field into the long junction. For the investigated case the characteristic frequency is $f_{c}=9.7 \mathrm{GHz} \approx \mathrm{f}_{\mathrm{LO}}$. A microwave penetration depth must be taken into account.

\section{FOUR JUNCTION INTERFEROMETER}

A long Josephson junction can be approximated by an interferometer with many point junctions. The circuit diagram of a long interferometer is sketched in Fig. 4. The microwave current is fed asymmetrically.

A simple approximation of a long Josephson junction is a four junction interferometer. Static gate characteristics of interferometers with many Josephson junctions are found in [10]. The static characteristic for a uniform interferometer with $\lambda=0.1 \pi$ and $N=4$ is plotted in Fig. 5a. Only the flux modes $\mathrm{M}=000,010,101$ and 111 exist for $0<\Phi_{\mathrm{C}}<\Phi_{0}$. The characteristic phase corresponds to an interferometer inductance of $\mathrm{L}=4.97 \mathrm{pH}$. The interferometer approximates a single long Josephson junction for $\Phi_{\mathrm{C}} \leq \Phi_{0} / 2$ with its normalized length

$$
\frac{l}{\lambda_{J}}=\sqrt{N \cdot(N-1) \cdot \lambda},
$$

where $\mathrm{N}$ is the number of Josephson Junctions in the interferometer. As the normalized length is $1 / \lambda_{\mathrm{J}}=1.9$, the interferometer approximates a long junction, where the self field effects can no more be neglected. Each curve 5b-d comprises 1200 points between $\Phi_{\mathrm{C}}=0$ and $\Phi_{\mathrm{C}}=3 \Phi_{0}$.

A microwave with an amplitude $\hat{I}_{L O}=0.2 \cdot I_{0}$ is below the threshold characteristic at every value of $\Phi_{\mathrm{C}}$. The peaks appear, if the microwave nearly reaches the threshold current. Three peaks are in each period $\mathrm{m}$ of $\Phi_{C}=m \cdot \Phi_{0}$. Equally large absorption maxima could be expected at the 1. and 3. peak in regarding only the static characteristic in Fig. 5a. However, the 3. peak is lower than the 1., because the microwave does not fully penetrate the interferometer. At

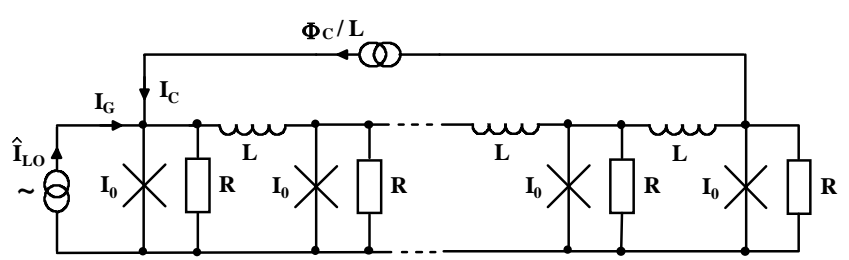

Fig. 4. Equivalent circuit of al long uniform interferometer with many equal Josephson-junctions. The microwave current is injected asymmetrically at the left end. The magnetic field is represented by a DC-current $\Phi_{\mathrm{C}} / \mathrm{L}$.

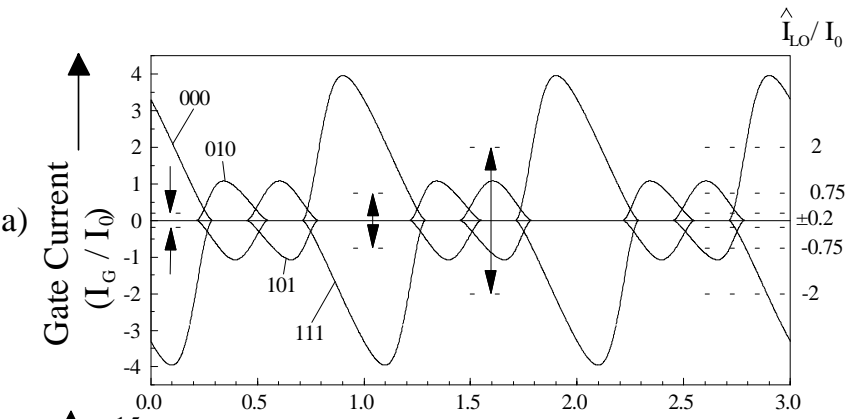

b)

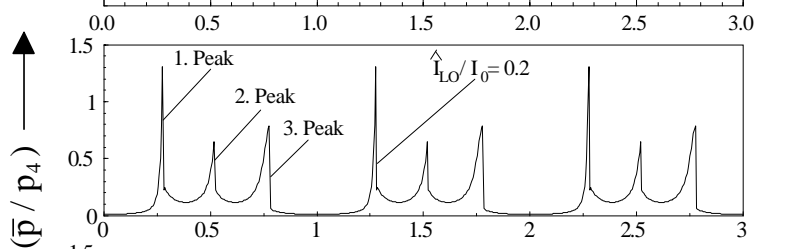

c)

d)

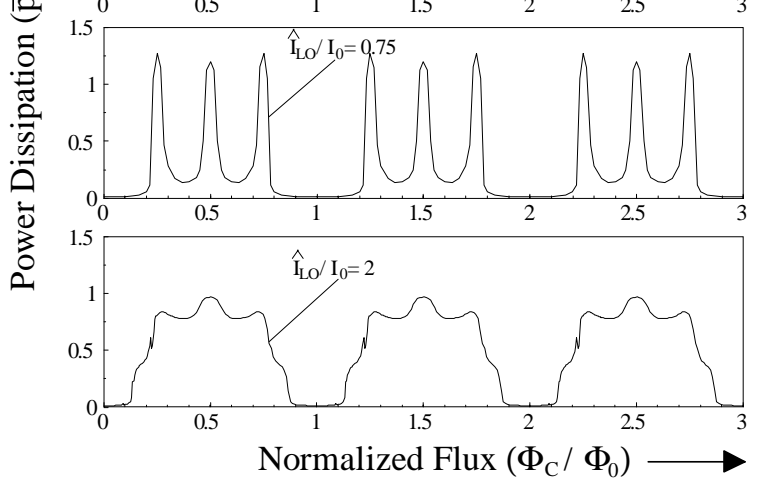

Fig. 5. Power dissipation of a four junction interferometer with $\lambda=0.1 \pi$, $1 / \lambda_{\mathrm{J}}=1.9$ and $\beta=1.3$.

a) Static interferometer characteristics. The flux modes 000,010 and 101 are stable.

Average power dissipation at the microwave amplitudes

$\begin{array}{lll}\text { b) } \hat{I}_{L O}=0.2 \cdot I_{0} & \text { c) } I_{L O}=0.75 \cdot I_{0} & \text { d) } I_{L O}=2 \cdot I_{0}\end{array}$

the 2. peak the microwave current does not reach the threshold current as close as in the 3 . peak, therefore the microwave absorption is smaller.

A microwave amplitude $\hat{I}_{L O}=0.75 \cdot I_{0}$ exceeds the threshold current. The simulation results are shown in Fig. 5c. The interferometer switches into the voltage state and the linewidth increases, too. In the voltage state the impedance of Josephson junctions changes and the microwave current can much better penetrate to the end of the interferometer. In this case all absorption peaks have the same maximum absorption. The influence of the flux modes $\mathrm{M}=010$ and 101 on the dissipation decreases with increasing microwave current as shown in Fig. 5d for $\hat{I}_{L O}=2 I_{0}$. A further increase of the amplitude yields a sinusoidal shape of the power dissipation versus flux.

To demonstrate a large microwave shielding effect, the inductance of an interferometer loop is increased to $\mathrm{L}=20 \mathrm{pH}$ corresponding to $\lambda=0.4 \pi$ and $1 / \lambda_{\mathrm{J}}=3.9$. The static threshold characteristics are plotted in Fig. 6a, where in contrast to the characteristic Fig. 5a the additional modes $\mathrm{M}=001,100,011$ and 110 are stable. They are within the modes 000, 010 and 101, so that the interferometer does not switch into the additional modes. The microwave current 


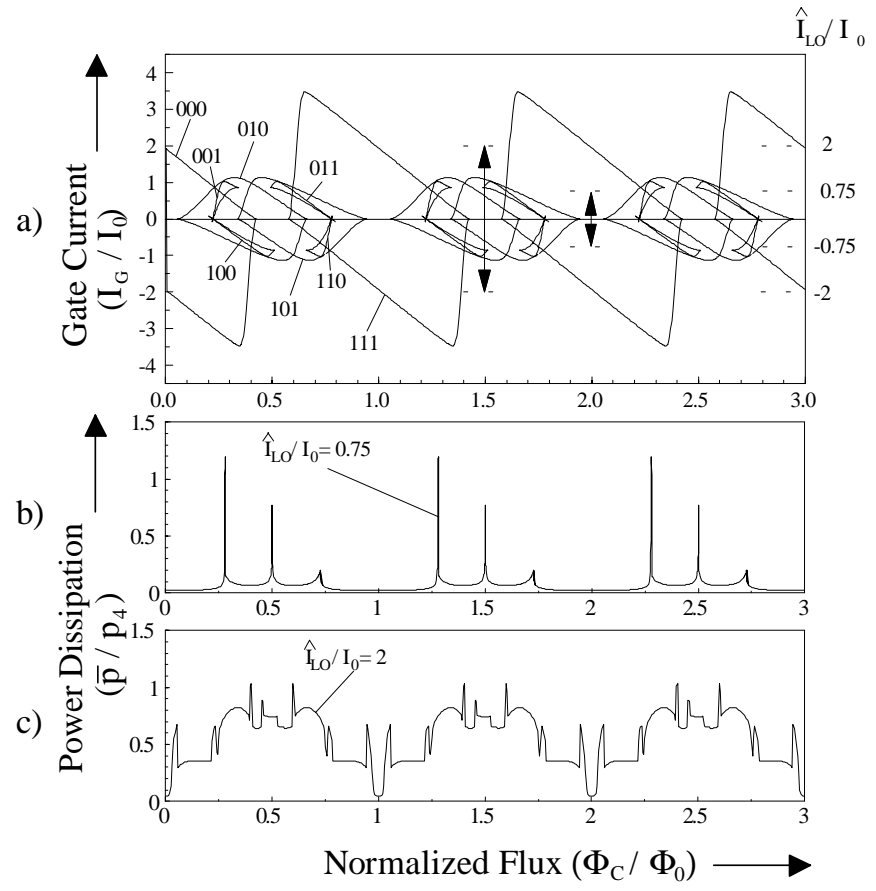

Fig. 6. Power Dissipation of a four junction interferometer with $\lambda=0.4 \pi$, $1 / \lambda_{\mathrm{J}}=3.9$ and $\beta=1.3$

a) Static interferometer characteristics, all possible flux modes are stable. Average power dissipation at the microwave amplitude

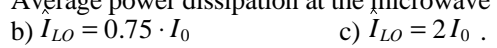

has been chosen below the static threshold.

The simulated result is shown in Fig. 6b. Peaks appear, if the microwave current switches the interferometer between two flux modes or gets close to the threshold of a flux mode. Similarly to the simulations with $\lambda=0.1 \pi$, a group of three peaks appears periodically with $\Phi_{C}=m \cdot \Phi_{0}$. The peak maximum within the group decreases with increasing normalized flux, because the microwave current is shielded by the interferometer inductance $\mathrm{L}$. To check the frequency dependence, the microwave frequency has been decreased to $1 \mathrm{GHz}$, while all other circuit parameters remained unchanged. At $\mathrm{f}_{\mathrm{LO}}=1 \mathrm{GHz}$ all peaks have the same maxima.

Absorption lines occur, when the phase of one or more Josephson junctions is close to $\varphi=\pi / 2$, i.e. the microwave current reaches the threshold. The closer the phase to $\pi / 2$ the higher will be the microwave absorption. The equivalent large nonlinear inductance of the Josephson junctions causes a high current through the damping resistor $\mathrm{R}$. If there is no flux quantum in the interferometer, the microwave current switches the junctions at the left end first. If there are more flux quanta trapped, the power dissipation depends on the inner junctions. If the microwave does not fully penetrate the interferometer, the junctions within the interferometer do not approach the threshold as far as for $f_{L O}<<f_{c}$. They strongly change, but do not switch into another mode, as in the quasistatic case.
For $\hat{I}_{L O} / I_{0}=2$ the interferometer enters the voltage state within each half cycle. Since the excursion of each Josephson junction phases gets larger than $2 \pi$ and since the characteristic phase is relatively large, each phase can have a more complicated dependence on time than for $\hat{I}_{L O} / I_{0}=0.75$. Therefore the absorption versus external field has a different character in Fig. $6 \mathrm{~b}$ and $\mathrm{c}$ especially in comparison with Fig. 5d. New lines appear in Fig. 6c. These new lines need further investigations.

\section{CONCLUSION}

Microwave absorption in a one dimensional array of strongly Josephson junctions and lumped inductances depend in a complicated way on the characteristic phase $\lambda$, the static threshold characteristic, the microwave current amplitude, the microwave and the characteristic frequency. Extremely small ranges of applied field exist, where the absorption has a sharp peak. The basic behavior of linewidth broadening is in agreement with measurements.

\section{ACKNOWLEDGEMENT}

The authors would like to thank C. Kessler, B. Nebendahl and Prof. M. Mehring for useful discussions.

\section{REFERENCES}

[1] H. Vichery, F. Beuneu, P. Lejay, "Microwave Absorption in Single Crystall of YBaCuO at Low Magnetic Field,"Physica C 159 (1989), pp 823-830

[2] T. K. Xia, D. Stroud, "Nonlinear electrodynamics and resonant microwave absorption in ceramic superconductors,"Phys. Rev. B, Vol. 39, No. 7, March 1989, pp 4792.4795

[3] H. Kratz, W. Jutzi, "Microscopic Simulation Model of Josephson Junctions for Standard Circuit Analysis Programs," IEEE Trans. Mag. Vol.-MAG 23, No. 2, March 1987, pp 731-734

[4] K. W. Blazey, A. M. Portis, K. A. Müller, J. G. Bednorz, F. H. Holtzberg, "Spin Glass Microwave Study in High- ${ }_{C}$ Superconductors," Intern. Conf. on High-Temp. Supercond. and Materials and Mechanism of Superconductivity, Interlaken, 1988

[5] K. W. Blazey, A. M. Portis, K. A. Müller, F. H. Holtzberg, "Macroscopic Flux Quantisation and Microwave Excitation in Single Crystal $\mathrm{YBa}_{2} \mathrm{Cu}_{3} \mathrm{O}_{7-\delta}$," Europhysics Letters, March 1989

[6] Y. Maniwa, A. Grupp, M. Mehring, "Mechanism of Direct Microwave Absorption in $\mathrm{YBa}_{2} \mathrm{Cu}_{3} \mathrm{O}_{7-\mathrm{x}}$," Physica C 156 (1988), pp 785-760

[7] H. Beha, "Asymetric 2 Josephson-Junction Interferometer as a Logic Gate," Electronics Letters, Vol. 13, No. 7, March 1977, pp 216-218

[8] J. Wunsch, W. Jutzi, E. Crocoll, "Parameter Evaluation of Asymmetric Interferometers with two Josephson-Junctions," IEEE Trans. Mag. , Vol. MAG-18, No. 2, March 1982, pp735-737

[9] C. Kessler, B. Nebendahl, A. Dulcic, Th. Wolf, M. Mehring, "Origin of discrete microwave absorptions lines in $\mathrm{YBa}_{2} \mathrm{Cu}_{3} \mathrm{O}_{7-x}$ single crystalls," Physica C 192 (1992), pp 79-84

[10] R. Simons, W. Jutzi, H. Beha, "Static Interferometer Characteristics with many equal Josephson junctions," IEEE Trans. Mag. Vol. MAG-19, No. 1, January 1983, pp 44-50 